\title{
Arthroscopic confirmation of femoral button deployment avoids post-operative X-ray in $\mathrm{ACL}$ reconstruction
}

Fabrizio Matassi , Giacomo Sani , Matteo Innocenti , Niccolò Giabbani \& Roberto Civinini

To cite this article: Fabrizio Matassi , Giacomo Sani , Matteo Innocenti , Niccolò Giabbani \& Roberto Civinini (2020): Arthroscopic confirmation of femoral button deployment avoids post-operative X-ray in ACL reconstruction, The Physician and Sportsmedicine, DOI: 10.1080/00913847.2020.1796469

To link to this article: https://doi.org/10.1080/00913847.2020.1796469

\section{Џ Article views: 13}

Accepted author version posted online: 15

Jul 2020.

Published online: 27 Jul 2020.

Submit your article to this journal

Q View related articles $\asymp$

View Crossmark data $₫$ 


\title{
Arthroscopic confirmation of femoral button deployment avoids post-operative X- ray in $A C L$ reconstruction
}

\author{
Fabrizio Matassi, Giacomo Sani $\mathbb{B}$, Matteo Innocenti, Niccolò Giabbani and Roberto Civinini \\ Orthopaedic Clinic, University of Florence, AOU Careggi, Florence, Italy
}

\begin{abstract}
Background: Anterior cruciate ligament reconstruction (ACLR) with cortical fixation adjustable-loop devices are associated with high potential risk of button malpositioning or interposition of the soft tissue between lateral femoral cortex and the button. Surgeons usually use X-rays to check and avoid button malposition and soft tissue interposition. Arthroscopic visualization of button position through the lateral gutter has been described. With this technique, it is possible for identification and correction of femoral button malalignment in the setting of soft tissue interposition and it could avoid the use of post-operative X-ray.

Methods: A total of 193 ACLR were included and patients were randomized into two groups. The first series (Group A) of 112 patients who sustained an ACLR with post-operative X-ray to assess the position of the femoral button and the second series (Group B) of 81 patients who sustained an ACLR with an arthroscopic exploration of the button followed by post-operative X-ray.

Results: On the post-operative radiographs, tissue interposition between the button and femoral cortex was found in nine cases of 112 in Group A (8\%) and in zero case of 81 in Group B (0\%). In six cases $(7,4 \%)$ in Group B, there was a soft tissue interposition between the button and femoral cortex as visualized by arthroscopic confirmation and before post-operative X-ray; in all these cases, the soft tissue was removed, and the button was in contact with the bone in all X-ray made in the Group B. Conclusions: This technique allows for identification and correction of femoral button malalignment in the setting of soft tissue interposition and reduces the use of post-operative $\mathrm{X}$-ray.
\end{abstract}

ARTICLE HISTORY

Received 24 April 2020 Accepted 13 July 2020

\section{KEYWORDS}

Knee ligament; button deployment; ACL; X-Ray

\section{Introduction}

Doubled semitendinosus and gracilis (DSTG) tendon autograft is the most common graft used for anterior cruciate ligament reconstruction (ACLR) [1]. This graft has spread in the last two decades for less graft-site morbidity and lower functional deficit compared to the patellar tendon (PT) graft [1]. However, DSTG requires tendon-to-bone healing with longer time for graft incorporation compared to bone-to-bone healing process required for PT. For this reason, a rigid fixation of DSTG graft is crucial especially during the early post-operative period and until graft incorporation has not been completed [2].

Cortical fixation with adjustable-loop or fixed-loop femoral suspension devices have demonstrated satisfactory biomechanical properties with high stiffness to restore stability of the knee and high resistance against slippage under cyclic loading conditions. Adjustable loop is provided with tensioning suture, that is located at the button end and allows to reduce the loop length allowing advancement of the graft into the femoral socket. In this way, there is a reduction of the need for multiple loops, increasing the amount of graft within the femoral tunnel available for incorporation with a reduction of the 'windshield wiper' effect $[3,4]$.

However, there are several case series documenting the potential complications of these devices, such as malpositioning and interposition of the soft tissue during fixation. In particular, the long loop of which adjustable devices are provided increases the risk to pull out the button far from the lateral cortex of the femur and flipping the button over the fascia or muscular tissue with consequent loss of graft tension. Mae et al. in their series reported an incidence of tissue interposition between the button and femoral lateral cortex in $25.2 \%$ of the cases with a consequent high risk of button migration at 1 year of follow-up [5].

To overcome these possible complications and ensure adequate placement of femoral button, the use of intra- or postoperative X-ray to confirm the button position against the lateral femoral cortex is generally in ACL reconstruction with DSTG $[5,6]$. However, there is a radiation exposition for the patients, increased time in the operating room, and risk of field contamination if performed intra-operatively. Arthroscopic exploration of the lateral gutter to ensure femoral button deployment is recently described with the aim to reduce soft tissue interposition and reduce the need for radiographic control [7-11]. Although the surgical technique has been reported by many authors, the real benefits of this procedure has not been well demonstrated.

The purpose of this study is to verify if direct arthroscopic or endoscopic visualization of the femoral button can identify malposition and assist in correction of malposition. The hypothesis is that arthroscopic or endoscopic visualization of 
the femoral button can identify malposition as well as intraoperative fluoroscopy or post-operative $\mathrm{X}$-ray.

\section{Patients and methods}

\section{Patients including description of the population}

We prospectively followed a series of 275 consecutive patients who underwent unilateral $A C L$ reconstruction from November 2015 to September 2018 at the author's institution. Inclusion criteria were as follows: age of more than 18 years, primary single-bundle $\mathrm{ACL}$ reconstruction using DSTG tendon graft, use of femoral cortical suspension (TightRope $\mathrm{RT}^{\mathrm{TM}}$ Arthrex Inc., Naples, Florida), and post-operative X-ray available to check button position. Exclusion criteria were as follows: previous surgery at the affected knee $A C L$ reconstruction with PT autograft, $A C L$ reconstruction using any allograft tissue, revision $A C L$ surgery, use of different types of femoral fixation from the cortical button, no post-operative X-rays available or properly done for measurement. After the application of the above inclusion and exclusion criteria, a total of 193 patients were included. Patients were divided into two groups. The first series (Group A) operated from November 2015 to March 2017 of 112 patients who sustained an $\mathrm{ACL}$ reconstruction with post-operative $\mathrm{X}$-ray to assess the position of the femoral button and the second series (Group B) operated from March 2017 and September 2018 of 81 patients who sustained an ACL reconstruction with an arthroscopic exploration of the button followed by post-operative X-ray. The two groups were comparable for age, sex, body mass index (BMI), and other demographic data (Table 1).

The institution approved the human protocol for this investigation; all investigations were conducted in conformity with ethical standards of the institutional and national research committee and with the 1964 Helsinki declaration and its later amendments. All patients have given their informed consent for participation and there is no financial interest to report.

\section{Surgical technique}

In all cases, the surgery was performed by one of the authors. Pre-operative antibiotic prophylaxis was administered with cefazolin $2 \mathrm{~g}$ i.v. or levoxacin $500 \mathrm{mg}$ i.v. in the case of penicillin allergy. After spinal anesthesia was performed the patient is placed in supine position and the tourniquet was applied at the proximal tight and inflated before the incision. Semitendinosus and gracilis tendon were harvested and prepared as a four-stranded double-looped autograft.

Table 1. Summary of the data collected. BMI (body mass index), international knee documentation committee (IKCD).

\begin{tabular}{lrrr}
\hline & \multicolumn{1}{c}{ Group A } & \multicolumn{1}{c}{ Group B } & p-value \\
\hline Numbre of patients & 112 & 81 & \\
Patient age (years) & 25.8 (range 18-37) & 26.7 (range 18-43) & 0.87 \\
Gender (M/F ratio) & 1.15 & 1.07 & 0.68 \\
BMI & 23.8 (range 19-32) & 22.6 (range 20-33) & 0.74 \\
Surgical time (minutes) & 63 (range 54-83) & 66 (range 53-92) & 0.45 \\
Lysholm Score & 97.2 (range 72-100) & 97.0 (range 62-100) & 0.83 \\
IKDC score & 75.6 (range 62-100) & 81.1 (range 57-100) & 0.75 \\
\hline
\end{tabular}

Standard anteromedial (AM) and anterolateral (AL) arthroscopic portals were used for arthroscopic exploration of the knee and any associated pathology related to the meniscus or cartilage were treated. An anatomic ACLR was then performed ensuring that femoral and tibial tunnel were created within the center of the native femoral and tibial footprint. The diameter of the tunnel was chosen equally to the diameter of the graft. The femoral tunnel was created using an outsidein technique with a retrograde drilling guide pin (Flipcutter ${ }^{T M}$ Arthrex Inc., Naples, Florida).

Anatomic tibial guide (Arthrex Tibial Guide Inc., Naples, Florida) was used to create a standard tibial tunnel. The graft was then passed through the tibial tunnel, across the joint, and into the femoral tunnel. The femoral fixation was performed using a cortical device (TightRope $\mathrm{RT}^{\mathrm{TM}}$ Arthrex Inc., Naples, Florida). The femoral button passage was controlled under arthroscopic visualization of the femoral tunnel and following the mark into the loop as suggested by the surgical technique [12]. Once the button is engaged into the lateral cortex of the femur, the tensioning suture is pulled to advance the graft into the femoral tunnel. The graft was tensioned and the tibial fixation was performed using an absorbable interference screw (BioComposite ${ }^{T M}$ Interference Screw Arthrex Inc., Naples, Florida) with the knee at $30^{\circ}$ of knee flexion. All patients received a post-operative $\mathrm{X}$-ray on the day of surgery to assess proper position for the femoral button (Figure 1).

Since March 2017, we introduced the arthroscopic exploration of the lateral gutter to confirm the femoral button deployment before graft tensioning. In the patient of Group $B$ before the advancement of the graft into the femoral tunnel, an exploration of the lateral gutter was performed. The knee is brought into extension and the scope is advanced into the lateral gutter through the AL portal. Lateral synovial recess is then gently cleaned using a sharp scissor introduced through the femoral lateral incision until the femoral button is visualized (Figure 2). The position of the button is checked for proper seating and soft tissue interposition between the button and cortex debridement was provided using a sharp instrument until the optimal button position is obtained. The remaining procedure was performed in a routine manner as described above.

\section{Evaluation}

Anteroposterior (AP) and latero-lateral (LL) radiographs were taken immediately after $\mathrm{ACL}$ reconstruction in the operating room. The AP radiographs were taken with the knee in slight internal rotation to avoid superimposition of the button with the lateral femoral cortex. The position of the button in the AP radiographs was graded as described by Toftoy et al.: 1) less than $2 \mathrm{~mm}$ of the distance between the whole button and the cortex (reduced and congruent); 2) more than $2 \mathrm{~mm}$ of the distance between the part of the button and the cortex (reduced and incongruent); 3) more than $2 \mathrm{~mm}$ of the distance between the whole button and the cortex (displaced); 4) part of the button within the bone (intraosseous) [13] (Figure 1). The $L L$ radiographs were used to confirm the position of the button anterior to the lateral supracondylar line [6]. Data were 


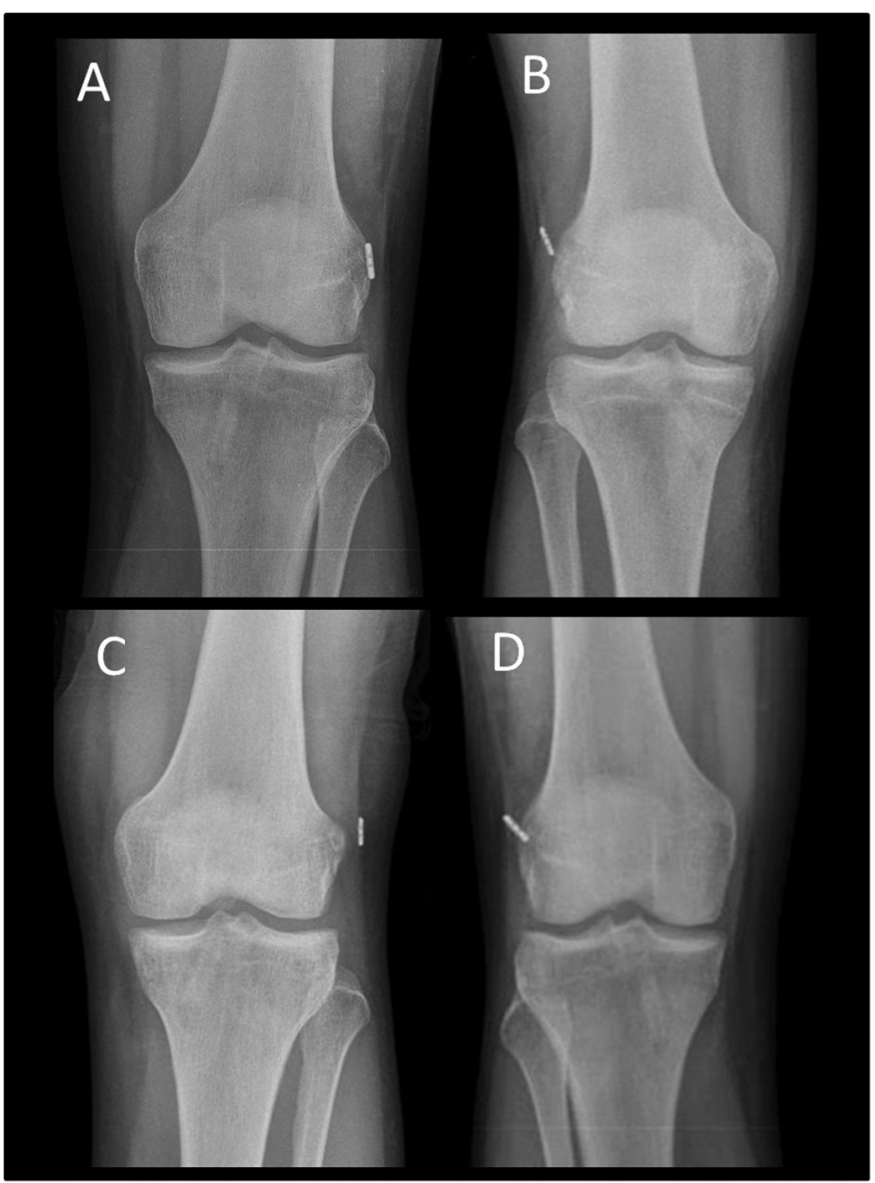

Figure 1. Classification of button position at anteroposterior radiographs. (a) Button reduced and congruent; (b) Button reduced and incongruent; (c) Button displaced; (d) Button intraosseous.

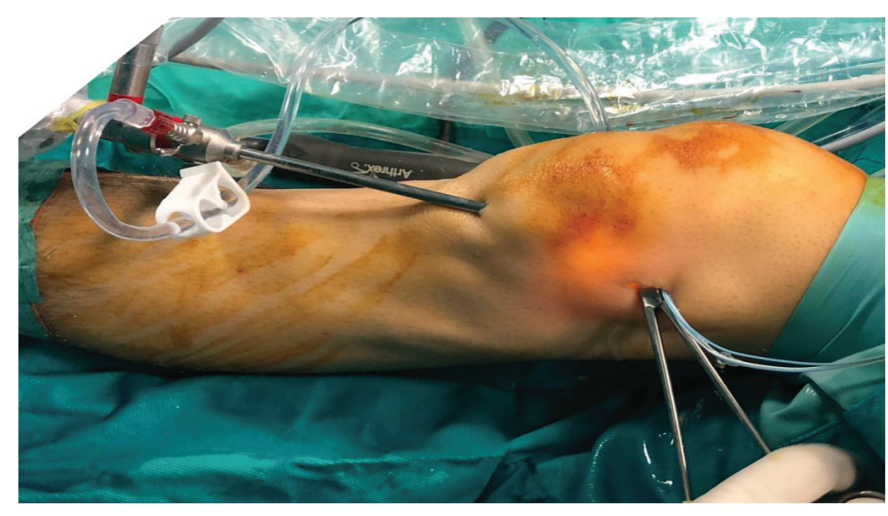

Figure 2. Arthroscopic exploration of the lateral gutter with the knee in full extension. The scope is advanced through the AL portal into the lateral synovial recess, and with a sharp scissor, soft tissue between the button and lateral cortex is removed.

recorded to an accuracy of $0.1 \mathrm{~mm}$ by two observers using a specialized software (CarestreamHelth, Rochester, NY).

Clinical evaluation was performed before surgery and at 6 months after surgery. Subjective assessment was performed using the International Knee Documentation Committee (IKDC) scores and Lysholm scores [14,15]. Side-to-side differences in AP laxity were measured using the KT-1000 ${ }^{\mathrm{TM}}$ arthrometer (MEDmetric Corp., San Diego, CA, USA) with 30-pound anteroposteriorly directed loads with the knee positioned in $25^{\circ}$ of flexion [16].

Surgical time was recorded in all the procedures. Complications were recorded in all the procedures.

\section{Statistical analysis}

Statistical analysis was performed using SPSS v19.0 (SPSS Inc., Chicago, Illinois) and Stata v13.0 (Stata Press, Lakeway Drive, College Station, TX, USA). All data were tested and found to be normally distributed using the KolmogorovSmirnov test. At 6 months, post-operative evaluation clinical improvement between the Groups A and B as described by the Lyshom score and IKDC as well as objective tibial anterior translation $(\mathrm{mm})$ described by the knee arthrometer measurements were analyzed using a t-test for paired comparisons. The Pearson correlation coefficient was used to assess whether a correlation exists between soft tissue interposition and clinical scores. A p-value of $<0.05$ was considered significant.

\section{Results}

On the post-operative radiographs, tissue interposition between the button and femoral lateral cortex was found in nine cases of 112 in the Group A (8\%) and in zero case of 81 in the Group B (0\%). Of nine cases of Group A with soft tissue interposition, six (5,35\%) were classified as reduced and incongruent, two (1,78\%) as displaced, and $1(0,89 \%)$ as intraosseous (Figure 1).

In six cases $(7,4 \%)$ in the Group $B$, there was a soft tissue interposition between the button and lateral femoral cortex as visualized by arthroscopic confirmation and before postoperative X-ray (Figure 3). Soft tissue was removed and proper seating of the button was obtained under direct arthroscopic control (Figure 4). In two cases in the Group B, there was an incomplete button deployment with the button in a vertical

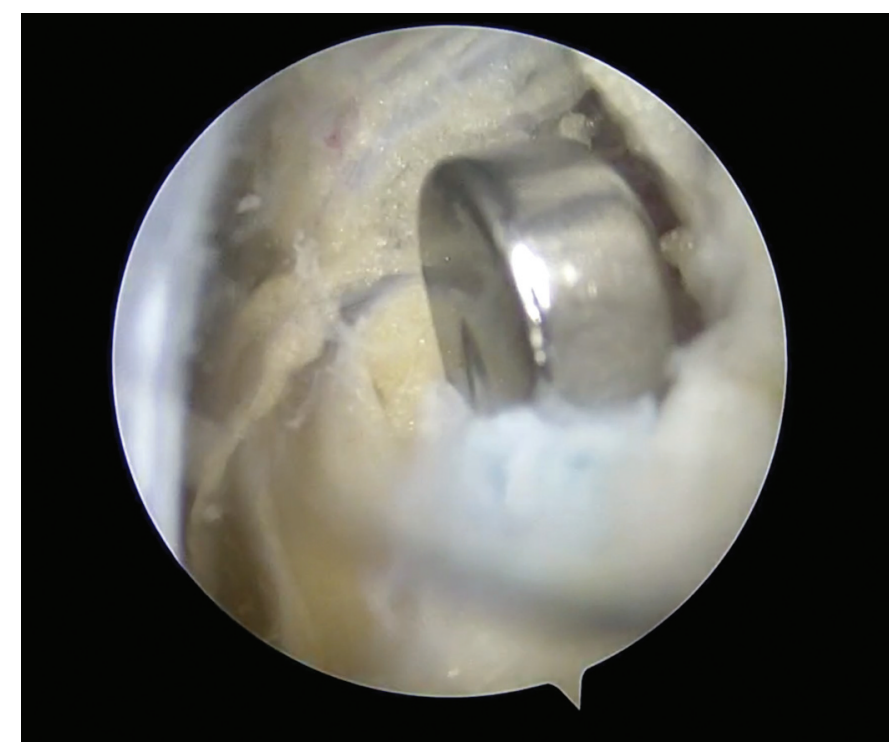

Figure 3. Soft tissue interposition between the button and lateral femoral cortex. 


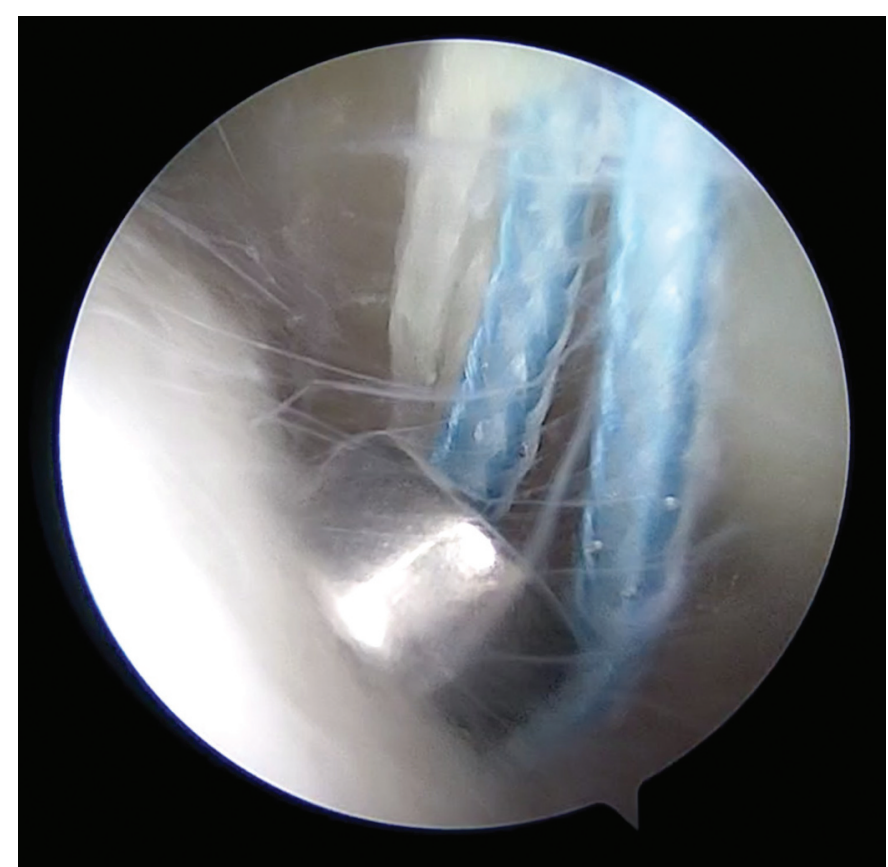

Figure 4. Proper seating of the button after soft tissue debridement.

position. A proper button seating was provided by pulling out the suture under direct arthroscopic visualization of the tilting process and cleaning the soft tissue around the button. No other intra-operative complications were observed, in particular, no complications were recorded related to the exploration of the lateral gutter. The mean surgical time was comparable $(p=0.87)$ between the two groups.

No post-operative thrombosis or infection was seen in the two groups. Hematoma formation was recorded in one case for Group A and two cases for Group B. Revision ACL for graft failure was performed in two patients in Group $A$ and in one patient in Group B after a subsequent trauma.

There was no significant difference in post-operative knee laxity at knee arthrometer between Group A and Group B at 6 months of follow-up $(2.11 \mathrm{~mm}$, SD $0.11 \mathrm{vs} .2 .64 \mathrm{~mm}$, SD 0.10, $\mathrm{p}=0.85$, ). No clinical differences were recorded regarding Lysholm score and IKDC score between the two groups at 6 months of follow-up. Clinical scores in the nine patients in the Group A with soft tissue interposition visualized in the post-operative X-ray were comparable to the clinical scores recorded in the other patients.

\section{Discussion}

Our findings suggested that arthroscopic confirmation of femoral button deployment is a safe procedure that reduces the risk of soft tissue interposition between the button and the lateral femoral cortex, ensuring proper seating of the femoral button without the use of X-ray. The use of intra- or postoperative $\mathrm{X}$-ray to confirm the button position against the lateral femoral cortex is generally used in ACL reconstruction with DSTG $[5,6]$. However, there is a radiation exposition for the patients, increased time in the operating room, and risk of field contamination if performed intra-operatively. Arthroscopic exploration of the lateral gutter to ensure femoral button deployment is recently described with the aim to reduce soft tissue interposition and reduce the need for radiographic control [11]. Although the surgical technique has been reported by many authors, the real benefits of this procedure have not been well demonstrated. The purpose of the current study is to verify if direct arthroscopic visualization of button deployment is effective in reducing the risk of button malposition and avoid the use of intra- or postoperative X-ray.

Adjustable-loop femoral cortical suspension device for ACLR with DSTG has demonstrated many technical advantages and is widely used [17]. However, there are some potential complications related to the long loop of these devices such as malposition of the button and soft tissue interposition between the button and the lateral femoral cortex $[5,13,18-20]$.

Toftoy et al. in a recent study demonstrated that in a series of 361 patients undergoing primary ACLR only 312 buttons (86.43\%) were reduced and congruent to the lateral cortex of the femur at the post-operative X-ray with a high rate of button malposition [13]. Similarly, Mae et al. described a rate of soft-tissue interposition between the femoral button and the lateral cortex up to $25 \%$ on post-operative radiographs after ACLR although clinical outcomes where not affected by button migration or soft tissue interposition at 1 year of follow-up [5]. However, soft tissue interposition weakens the femoral fixation with the potential risk of graft loosening or migration of the button [6].

Perumal et al. modified this technique with the knee in full extension in order to relax the lateral soft tissue and obtain better visualization of the lateral gutter [8]. In our experience, we found advantages in performing button exploration with the knee in full extension allowing better visualization of the button deployment and better removal of the soft tissue around the button. Patel et al. reported a reduction of complication related to the femoral fixation with a button using an arthroscopic technique for exploration of the femoral tunnel through the AM portal providing direct visualization of the button passage and engagement of the lateral femoral cortex [9].

However, the aforementioned study reported technical notes or personal experiences. As far as we are concerned, no reports have demonstrated the real benefits of these procedures during $A C L R$ in the reduction rate of button malposition. From our findings, we can affirm that the arthroscopic exploration of the button deployment is a safe procedure and it is effective in reducing the risk of the button malposition. Moreover, this procedure allows the surgeons to easily remove soft tissue between the button and the femoral cortex providing proper deployment of the button.

Some limitations were identified and need to be considered when interpreting these data. First, the relative small series of this study is underpowered to detect the meaningful clinical difference between the two groups. Nonetheless, the focus of the present study was to analyze the proper position of the femoral button in the immediate post-operative, rather than presenting clinical outcomes at long-term follow-up. However, this technique allows to check proper seating of the femoral button against lateral 
cortex of the femur and it could be used for any femoral suspensory device with cortical button fixation.

\section{Conclusions}

Arthroscopic confirmation of the femoral button deployment is a safe technique as no complications were recorded and easy to use with no learning curve for surgeon confident with arthroscopic procedures. This technique allows the identification and correction of femoral button malalignment in the setting of soft tissue interposition and avoids the use of postoperative X-ray.

\section{Level of evidence}

Level III

\section{Declaration of interest}

The authors declare that they have no conflict of interest.

\section{ORCID}

Giacomo Sani (iD http://orcid.org/0000-0002-0445-5446

\section{References}

1. Kousa $P$, Järvinen $T L$, Vihavainen $M$, et al. The fixation strength of six hamstring tendon graft fixation devices in anterior cruciate ligament reconstruction. Part I: femoral site. Am J Sports Med. 2003;31:174-181.

2. Brand J Jr, Weiler A, Caborn DN, et al. Graft fixation in cruciate ligament reconstruction. Am J Sports Med. 2000;28:761-774.

3. Bedi A, Kawamura S, Ying $L$, et al. Differences in Tendon graft healing between the intra-articular and extra-articular ends of a bone tunnel. Hss J. 2009;5:51-57.

4. Giorgio N, Moretti L, Pignataro $\mathrm{P}$, et al. Correlation between fixation systems elasticity and bone tunnel widening after $\mathrm{ACL}$ reconstruction. Muscles Ligaments Tendons J. 2016;6:467-472.

5. Mae T, Kuroda S, Matsumoto N, et al. Migration of endobutton after anatomic double-bundle. Arthroscopy. 2011;27:1528-1535.

6. Uchida R, Mae T, Matsumoto N, et al. The effect of cortical button location on its postoperative. Knee Surg Sports Traumatol. 2014:22:1047-1054.
7. Sonnery-Cottet B, Rezende FC, Martins Neto A, et al. Arthroscopically confirmed femoral button deployment. Arthrosc Tech. 2014;2014(3):309-312.

8. Perumal R, Gunasekaran C, Jacob M, et al. Alternate method of arthroscopically confirming femoral button deployment for knee anterior cruciate ligament graft suspensory cortical fixation. Arthrosc Tech. 2018;7:1295-1298.

9. Patel SD, Boxley PJ, Kang RW. Arthroscopic anterior cruciate ligament femoral tunnel visualization for button fixation. Arthrosc Tech. 2017;6:585-589.

10. Nag HL, Gupta H. Seating of tightRope RT button under direct arthroscopic visualization in anterior cruciate ligament reconstruction to prevent potential complications. Arthrosc Tech. 2012;1:83-85.

11. Ohnishi $Y$, Chang A, Utsunomiya $H$, et al. Arthroscopic technique to reduce suture button migration during anterior cruciate ligament reconstruction procedure. Arthrosc Tech 2017;6:1927-1931.

12. Lubowitz JH, Ahmad CS, Anderson K. All-inside anterior cruciate ligament graft-link technique: second-generation, no-incision anterior cruciate ligament reconstruction Arthroscopy. 2011;27:717-727.

13. Toftoy AC, Rud CT, Deden AA, et al. Femoral cortical button malposition rates in anterior cruciate ligament reconstruction a retrospective review. Orthopedics. 2019;42:56-60.

14. Irrgang JJ, Anderson AF, Boland AL, et al. Development and validation of the international knee documentation committee subjective knee form. Am J Sports Med. 2001;29:600-613.

15. Lysholm J, Gillquist J. Evaluation of knee ligament surgeryresults with special emphasis on use of a scoring scale. Am J Sports Med. 1982;10:150-154.

16. Daniel DM, Stone ML, Sachs $R$, et al. Instrumented measurement of anterior knee laxity in patients with acute anterior cruciate ligament disruption. Am J Sports Med 1895;13:401-407.

17. Boyle MJ, Vovos TJ, Walker CG, et al. Does adjustable-loop femoral cortical suspension loosen after anterior cruciate ligament reconstruction? A retrospective comparative study. Knee. 2015;22:304-308.

18. Simonian PT, Behr CT, Stechschulte DJ Jr, et al. Potential pitfall of the endoButton. Arthroscopy. 1998;14:66-69.

19. Muneta T, Yagishita K, Kurihara Y, et al. Intra-articular detachment of the endobutton more than 18 months after anterior cruciate ligament reconstruction. Arthroscopy. 1999;15:775-778.

20. Yanmis I, Tunay S, Oguz E, et al. Dropping of an endoButton into the knee joint 2 years after anterior cruciate ligament repair using proximal fixation. Arthroscopy. 2004;20:641-643. 